\title{
Pulsed Gas Introduction into Quadrupole Ion Traps
}

\author{
W. Bart Emary, ${ }^{*}$ Raymond E. Kaiser, Hilkka I. Kenttämaa, and \\ R. Graham Cooks \\ Department of Chemistry, Purdue University, West Lafayette, Indiana, USA
}

Two different Paul-type quadrupole ion traps were cquipped with pulsed-valve gas inlets. The duration of a gas pulse inside the trap is variable, and pulses as short as $50 \mathrm{~ms}$ (FWHH) have been measured, allowing the use of several gas pulses during one experiment. The benefits of pulsed valves are outlined and demonstrated for chemical ionization experiments and for the use of selective ion-molecule reactions in structure determination of ions and neutral molecules. (] Am Soc Mass Spectrom 1990, 1, 308-311)

$\mathrm{T}$ The unique capabilities that are characteristic of trapped-ion mass spectrometry [1] include the ability to carry out multiple-stage experiments involving consecutive dissociation and/or ion-molecule reactions [2]. Endothermic and exothermic reactions can be studied, and the dependence of reactions on pressure, time, and energy can readily be investigated $[1,3]$. These features allow detailed studies of structures, reactions, and thermochemistry of many ionic and neutral gas-phase species, including those that are difficult or impossible to study in solution owing to their high reactivity.

Tandem mass spectrometry (MS/MS) experiments carried out in ion-trapping devices are pulsed in nature. In these single-region mass spectrometers, ionization, mass selection, reactions, and mass analysis occur in the same space and are separated in time, in contrast to the spatial separation employed in conventional mass spectrometers. Unwanted ions can usually be removed from the reaction region by applying appropriate voltage pulses to the trap. However, the same does not apply to neutral molecules. It follows that interfering reactions are not uncommon in trapped-ion mass spectrometry; for example, massselected ions may react faster with their neutral precursors than with the desired neutral reagents.

Pulsed-valve sample introduction was first introduced [4] for ion cyclotron resonance (ICR) traps to allow time resolution for neutral reagents. This allowed many chemical reactions requiring high pressure to be carried out while satisfying the high-vacuum requirement $\left(\leq 10^{-9}\right.$ lorr) for analysis of ions present in the trap. We report here the application of pulsedvalve technology for a quite different ion-trapping de-

\footnotetext{
* Present address: Department of Pharmacology, The Johns Hopkins University, $725 \mathrm{~N}$. Wolfe Street, Baltimore, MD 21205.

Address reprint requests to Hilkka I. Kenttämaa, Department of Chemistry, Purdue University, West Lafayette, IN 47907
}

vice, the Paul-type quadrupole ion trap. In this device, ions are stored in a three-dimensional quadrupole field formed between three electrodes with hyperbolic inner surfaces [5]. In contrast to the ICR technique, which uses a stationary electric field and a strong magnetic field to trap the ions, quadrupole ion traps use purely electrostatic confinement of ions. Quadrupole ion traps can operate [6] under significantly higher pressures (up to $10^{-3}$ torr) than ICR instruments, and the use of relatively high pressures of reagent gases is therefore not a problem. However, we will demonstrate here that pulsed-valve reagent introduction provides a simple means to enhance control over the reactions occurring in the trap. This is especially important for those quadrupole ion traps that do not have the capabilities necessary for single-ion isolation.

\section{Experimental}

Two different quadrupole ion traps were employed in this study, a Finnigan ion trap detector with modified [7] sample inlet system, and a prototype Finnigan ion trap mass spectrometer (ITMS) [8]. These instruments are pumped with a $50 \mathrm{~L} / \mathrm{s}$ and a $170 \mathrm{~L} / s$ turbomolecular pump, respectively, and the base pressure in both devices is in the low $10^{-7}$ torr range. A modified version of the commercial software was used in both instruments $[7,8]$. Ions were generated by electron ionization (EI) (typically for $1 \mathrm{~ms}$ ). The sequence of radiofrequency ( $\mathrm{rf}$ ) and direct current (dc) voltages used in the experiments described here are shown in Figure 1 for the ITMS; the same pulse sequence applies to the ion trap detector except that no dc voltages are used in this device. Typically, the ion isolation time was 4-7 ms, and the reaction time was varied from 0 to $1600 \mathrm{~ms}$. The amplitude of the if voltage applied at 1.1 $\mathrm{MHz}$ to the ring electrode determines the mass range of the ions trapped. Single-ion isolation and collisionactivated dissociation (CAD) experiments can be car- 


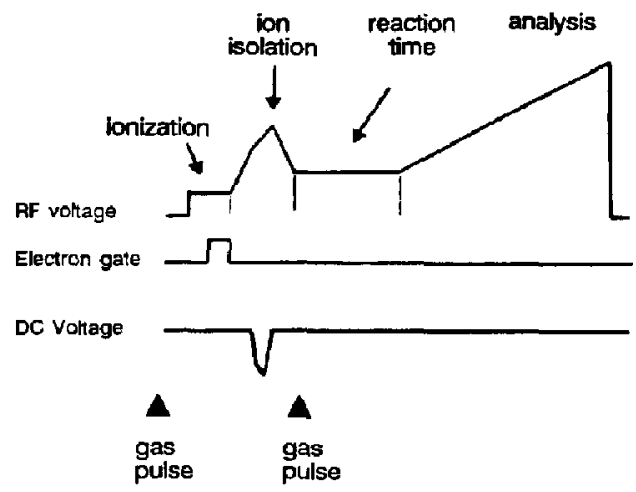

Figure 1. The sequence of radio-frequency (rf) and direct current (dc) voltages used in the quadrupole ion traps to generate ions (ionization), mass select the ion of interest (ion isolation), store the ionic products formed during ion-molecule reactions (reaction time), and analyze the ionic products (analysis). A typical EI time was $0.5 \mathrm{~ms}$ and ion isolation time $4-7 \mathrm{~ms}$; the reaction time was varied from 0 to $1600 \mathrm{~ms}$. The arrows indicate the times when the pulsed valve was opened in different experiments.

ried out by using the ITMS [8]. Single-ion isolation is accomplished by raising the rf voltage level (i.e., by raising the $q$ value) and then applying an appropriate dc voltage to the ring electrode (time interval typically $7 \mathrm{~ms}$ ). In the ion trap detector, only the ion(s) with the highest mass-to-charge $(\mathrm{m} / \mathrm{z})$ ratio in the trap in each experiment can be cleanly mass-selected. This is carried out by raising the rf level to eliminate all lighter ions from the trap by using an rf ramp only [7]. Prior to the reaction period, the $\mathbf{r f}$ level is lowered in both instruments in order to trap all product ions formed. After the reaction period, the ionic products are ejected from the ion traps by using mass-selective instability, controlled by ramping the amplitude of the if voltage, and detected with an external electron multiplier.

A General Valve Corporation Series 9 pulsed valve [4] was installed in both quadrupole ion traps. A commercial high-voltage power supply and a driver unit (IOTA 1, General Valve Corporation) were used to operate the pulsed valves. Materials inside the valve are Teflon and stainless steel. The exit orifice of the valves used is 0.006 in. in diameter. The duration of the open period is from $160 \mu \mathrm{s}$ to an indefinitely long time. The gas exits the valve into 1/16-in. stainless steel tubing $(\sim 3 \mathrm{~cm})$, connected via a swagelock adapter and 1/4in. tubing to the vacuum manifold of the ion traps. Gas flow was directed axially into the ion trap detector and equatorially into the ITMS. A trigger pulse initiated by the ion trap software was used to control the pulsing rate. Except for a pulse-conditioning circuit between the trigger output and the power supply input, no instrumental modifications were necessary. The ion trap software was modified to allow pulsing of the valve at different times during the experiment. Arrows in Figure 1 indicate the times selected for the present work: the gas pulse was initiated either at the beginning of the pre-ion time (a time interval prior to ionization time) or at the beginning of the reaction time. It is useful to have a relatively long delay between scans for complete pumpout of the pulsed gas ( $\geq 100 \mathrm{~ms}$ ).

The samples were obtained commercially. Unless otherwise specified, they were introduced into the vacuum manifold of the ion traps through GranvillePhillips leak valves at a nominal pressure of $(1-5) \times 10^{-6}$ torr, as measured with an ionization gauge in the vacuum manifold. The nominal pressure of the helium buffer gas was $1 \times 10^{-4}$ torr. Experiments carried out without the helium buffer gas yielded results similar to those obtained with the buffer gas.

\section{Results and Discussion}

For the configurations studied, the pulsed gas enters the trap within a few milliseconds after the valve is opened. In the absence of helium buffer gas, the peak pressure in the trap was in the $10^{-7}$ torr range, and this was reached when the valve had been open for $150-300 \mu \mathrm{s}$. The inlet pressure to the valve was 200 mtorr. The duration of a typical gas pulse inside the trap was measured for two different gases, isobutane and air, with and without the helium buffer gas. This was carried out by monitoring the ion intensities generated by EI of the gases at variable delay times after the gas pulse, with all other variables remaining constant. Figure 2 shows the concentration of $\mathrm{N}_{2}$ pulsed into the ion trap detector (no helium buffer gas) and measured as $\mathrm{N}_{2}+$ as a function of time. The shape of the gas pulse resembles that obtained earlier for ICR traps but appears to be somewhat narrower [4]. The pulse width depends on the exact location of the valve. Shortening the connecting stainless steel tube from about $25 \mathrm{~cm}$ to $2.5 \mathrm{~cm}$ decreased the pulse width (FWHH) from over $100 \mathrm{~ms}$ to less than $50 \mathrm{~ms}$ for the ion trap detector. The use of helium buffer gas did not appear to affect the pulse width.

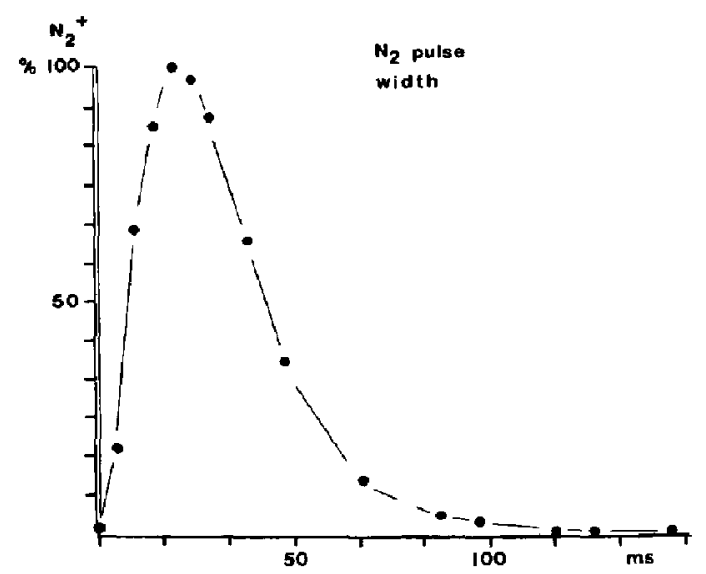

Figure 2. The variation of the $\mathrm{N}_{2}+$ signal intensity in the ion trap detector as a function of time from opening the pulsed valve to introduce $\mathrm{N}_{2}$. No helium buffer gas was used for this experiment. 


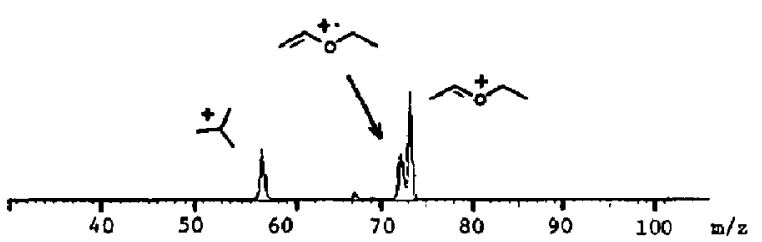

(a)

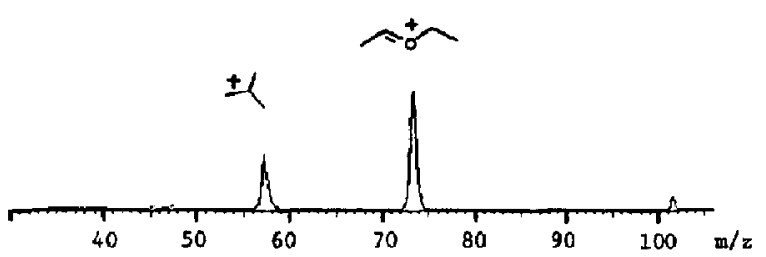

(b)

Figure 3. Isobutane $\mathrm{CI}$ of ethyl vinyl ether in the ion trap detector. (a) Constant pressures of the isobutane reagent gas and ethyl vinyl ether result in abundant tert-butyl cation ( $m / z 57$ ), protonated ethyl vinyl ether $(m / z 73)$, and ethyl vinyl ether radical cation $(m / z 72)$. (b) Fixed pressure of isobutane with pulsed introduction of ethyl vinyl ether. Isobutane was ionized during the ionization time to give tert-butyl cation $(\mathrm{m} / \mathrm{z} 57)$. Ethyl vinyl ether was introduced into the trap at the beginning of the reaction time.

The shortest pulse width accessible $(<50 \mathrm{~ms}$ FWHH) is quite narrow compared to the duration of the entire scan sequence of the ion trap (typically $500-1500 \mathrm{~ms}$ ). Therefore, it is possible to use several gas pulses during different stages of a single experiment. Moreover, it is feasible to have a pulse repetition rate of $100 \mathrm{~ms}$ with complete clearing of prior gases and without buildup of pressure in the trap. By placing the valve in a gas line between the trap and a metering valve, it was possible to use the line either for pulsed operation or for stationary pressure operation and rapidly switch between these two modes.

The use of pulsed reagent and/or sample introduction offers a valuable means to enhance control over chemical ionization (CI) experiments. Figure 3 shows two different experiments carried out to protonate ethyl vinyl ether by using isobutane reagent gas in the ion trap detector. For Figure 3a, constant pressures of isobutane and ethyl vinyl ether were used throughout the entire experiment. Some protonation of ethyl vinyl ether occurs to yield ions of $m / z 73$. However, the molecular ion of ethyl vinyl ether $(\mathrm{m} / \mathrm{z}$ 72) is also rapidly formed. This results at least partially from direct EI of ethyl vinyl ether and is difficult to avoid without using an extremely low partial pressure of the sample. For the spectrum shown in Figure $3 b$, ethyl vinyl ether was pulsed in the trap after generation of tert-butyl cation $(m / z 57)$ by EI of isobutane. Clean and quantitative protonation of ethyl vinyl ether is achieved. No interference from radical cation chemistry is indicated in this experiment.

Examination of the presence of specific functional groups in sample molecules by using selec- tive ion-molecule reactions provides another example where pulsed gas introduction is advantageous. An interesting reaction reported recently [9] involves formal transfer of acetylene from ethyl vinyl ether to protonated analyte if the analyte contains a $\beta$ hydroxycarbonyl functionality. In the present study, the ion trap detector was used to examine the reaction of protonated 4-hydroxy-3-methyl-2-butanone (analyte) with ethyl vinyl ether. When constant pressures of the analyte and the reagent ethyl vinyl ether were employed, protonated ethyl vinyl ether $(m / z 73)$ and its reaction products (mainly ethylated ethyl vinyl ether [10], $m / z$ 101) dominated the product distribution. Because of its high basicity [11], ethyl vinyl ether readily deprotonates all ions (including the protonated analyte) formed during and after EI of the mixture of ethyl vinyl ether and 4-hydroxy-3-methyl-2-butanone. By protonating the $\beta$-hydroxycarbonyl compound first $(m / z$ 103; Figure 4a), and later pulsing ethyl vinyl ether into the trap (Figure 4 b), it was possible to identify unambiguously the product ions resulting from reactions of the protonated analyte with ethyl vinyl ether. These are protonated ethyl vinyl ether $(m / z$ 73) and vinylated 4-hydroxy-3-methyl-2-butane $(\mathrm{m} / \mathrm{z}$ 129). The ion of $m / z 129$ indicates the presence of $\beta$ hydroxycarbonyl in the analyte.

In the examples discussed above, the use of pulsed valves allowed generation of a CI reagent without interference from the analyte, and ionization of the analyte without interference from the neutral reagent needed later in the experiment. Both experiments were carried out in an instrument that does not have singleion isolation capabilities. While these two experiments could be accomplished without using pulsed valves in an instrument that allows single-ion isolation, this is not true in cases where the ions of interest rapidly

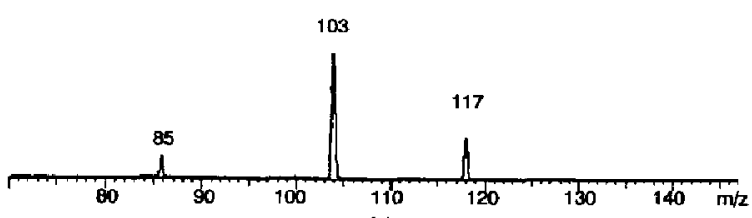

(a)

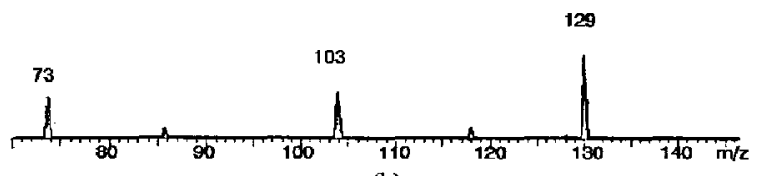

(b)

Figure 4. Identification of a specific arrangement of functional groups in an ionized analyte by a selective ion-molecule reaction in the ion trap detector. (a) Self-CI of the analyte, 4-hydroxy-3methyl-2-butanone (MW 102) (100 ms reaction time). The ion of $m / z 85$ is the dehydration product of the protonated analyte; the ion of $m / z 117$ arises from an impurity in the sample. (b) The same experiment as in (a) except that ethyl vinyl ether reagent was pulsed into the trap at the beginning of the reaction time. Addition of acetylene to the protonated analyte (to give $m / z 129$ ) indicates the presence of the $\beta$-hydroxycarbonyl functionality. 


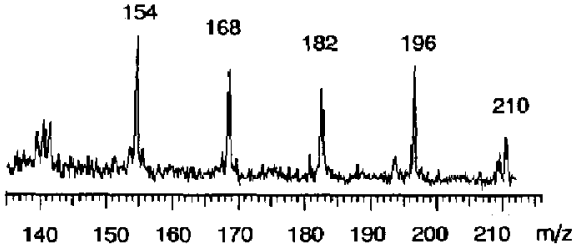

(a)

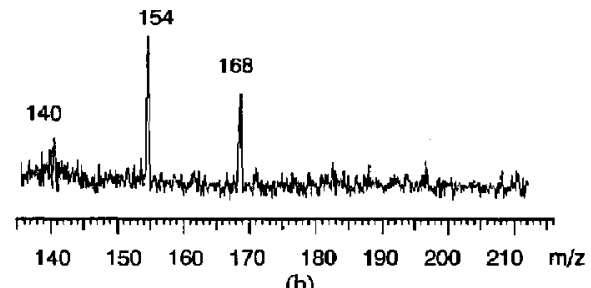

(b)

Figure 5. Location of carbon-carbon double bonds in long-chain alkenes by selective ion-molecule reactions in the ITMS. (a) Constant pressures of the analyte 2-hexene and the reagent gas 4 nonene were employed. After EI, the molecular ion of 4-nonene $(m / z$ 126) was isolated, and it reacted with the two alkenes present in the trap. Ionized 4-nonene reacts with neutral 4 nonene to give the ions of $m / z 182,196$, and 210 and with neutral 2-hexene to give the ions of $m / z$ 140, 154, and 168. (b) Constant pressure of the analyte was used. 4-Nonene was pulsed into the trap before the ionization time, and the molecular ion of 4-nonene was generated. Neutral 4-nonene was then pumped from the trap, and ionized 4-nonene was isolated and allowed to react with the analyte. The product ion peaks at $m / z$ values 140,154 , and 168 indicate that the analyte is 2-hexene. No major products were formed in either experiment outside the mass range shown

react with their neutral precursors. We recently examined [12] the possibility of using ion-molecule reactions involving an ionized alkene with known structure to locate the positions of carbon-carbon double bonds in unknown alkenes. If two different alkenes are present in an ion trap at the same time, however, the product distribution is likely to be complex, as is seen in Figure 5a, where reactions of ionized, isolated 4nonene with neutral 4-nonene compete with reactions of the analyte, neutral 2-hexene. A more successful approach involves pulsing the neutral reagent (4-nonene) into the ion trap and allowing enough time for the neutral 4-nonene to be pumped away prior to isolation of ionized 4-nonene and examination of its reactions with the analyte. In this experiment (Figure 5b), only products characteristic [12] of reaction of ionized 4-nonene with 2-hexene are obtained (ions of $m / z 140,154$, and 168).

\section{Conclusions}

Pulsed solenoid valves can be used for gas introduction into Paul-type quadrupole ion traps with minimal modification of software and hardware. Pulsed introduction of neutral reagents enhances control over jon-molecule reactions occurring in the trap. This will be especially valuable for those trapped-ion devices that do not have the capabilities necessary for singleion selection. The pulsed gas inlet methodology (1) provides a greater selection of reactant ions for CI experiments, (2) allows studies of ions that rapidly react with their neutral precursors, and (3) facilitates studies concerning mechanisms of ion-molecule reactions. Furthermore, the ability to rapidly change the type of neutral molecules in the trap allows practically simultaneous measurement of CI spectra using different reagent gases and the examination of $C A D$ at the same time as ion-molecule reactions. Finally, CAD experiments are likely to greatly benefit from the use of pulsed gas introduction. Not only will pulsed collision gas introduction allow the use of target gases other than the helium buffer gas without compromising the overall performance of quadrupole traps, but also pulsed sample introduction will prevent problems caused by interfering ion-molecule reactions, such as disappearance of CAD products through ion-molecule reactions with the neutral sample molecules.

\section{Acknowledgments}

Financial support provided by the National Science Foundation is gratefully acknowledged (HIK: grant CHE-8717380; RGC: grant CHE-8721768). We thank Jacques Einhorn for help in some experiments.

\section{References}

1. See, for example: (a) Laude, D. A., Jr.; Johlman, C. L.; Brown, R. S.; Weil, D. A.; Wilkins, C. L. Mass Spectrom. Rev., 1986, 5, 106-166. (b) Nourse, B.: Cooks, R. G. Anal. Chim. Acta 1990, 228, 1-21.

2. See, for example: (a) Cody, R. B.; Burnier, R. C.: Cassady, C. J.; Freiser, B. S. Anal. Chem. 1982, 54, 2225-2228. (b) Louris, J. N.; Brodbelt-Lustig, J. S.; Cooks, R. G.; Glish, G. L.; Van Berkel, G. J.; McLuckey, S. A. Int. J. Mass Spectrom. Ion Processes 1990, 96, 117-137.

3. Wanczek, K. P. Int. J. Mass Spectrom. Ion Processes 1984, 60, 11-60.

1. Carlin, T. J.; Freiser, B. S. Anal. Chem. 1983, 55, 571-574.

5. (a) Todd, J. F. J.; Lawson, G.; Bonner, R. G. In Quadrupole Mass Spectrometry; Dawson, P. H., Ed.; Elsevier: New York, 1976; Chapter 8. (b) March, R. E.; Hughes, R. J.; Todd, J. F. J. Quadrupole Mass Spectrometry; Chemical Analysis Series, Vol. 102; Wiley: New York, 1989.

6. Stafford, G. C.; Kelley, P. E.; Syka, J. E. P.; Reynolds, W. E.; Todd, J. F. J. Int. J. Mass Spectrom. Ion Phys. 1984, 60, 85-98.

7. Brodbelt, J. S.; Louris, J. N.; Cooks, R. G. Antal. Chem. 1987, $59,1278-1285$.

8. Louris, J. N.; Cooks, R. G.; Syka, J. E.; Kelley, P. F.; Stafford G. C.; Todd, J. F. J. Anal. Chem, 1987, 59, 1677-1685.

9. Kenttämaa, H. I.; Cooks, R. G. I. Am Chem. Soc. 1989, 111, 4122-4123.

10. Kenttamaa, H. I.; Pachuta, R. R.; Rothwell, A.; Cooks, R. G. J. Am. Chem. Soc. 1989, 111, 1654-1655.

11. Lias, S. G.; Bartmess, J. E.; Liebman, J. F.; Holmes, J. L.; Levin, R. D.; Mallard, W. G. J. Phys. Chem. Ref. Data 1988, 17, Suppl. 1.

12. Einhorn, J.; Kenttämaa, H. I.; Cooks, R. G. submitted for publication. 